\title{
Destruction of Bose-Einstein condensate by strong interactions
}

\author{
Mariusz Gajda, Magdalena A. Załuska-Kotur, and Jan Mostowski \\ Instytut Fizyki PAN $\&$ College of Science, \\ Aleja Lotników 32/46, 02-668 Warszawa, Poland
}

\begin{abstract}
We study exactly soluble system of trapped bosonic particles interacting by a model harmonic forces. The model allows for detailed examination of the order parameter (condensate wave function) as well as concept of the off-diagonal and diagonal order. We analyze the effect of interactions on the condensate and show that sufficiently strong interactions, attractive or repulsive, lead to destruction of the condensate. In the thermodynamic limit this destruction has a critical character. It is shown that the existence of the coherent state of bosons is related to existence of two length scales determined by one- and two-particle reduced density matrices. The condensate can exist only if the two length scales are of the same order. Interactions, both repulsive and attractive, change their relative size which may lead to destruction of coherence in the system and depletion of the condensate. We suggest that this scenario is model independent.
\end{abstract}

PACS number(s): 03.75.Fi, 05.30.Fk

\section{INTRODUCTION}

Recent advances in the trapping and cooling techniques which led to the achievement of the Bose-Einstein condensation of dilute gases have renewed interest in various aspects of many body theory. A cloud of weakly interacting trapped atoms is an ideal system for which various aspects of many body theory can be tested and verified. The ideal bosonic gas undergoes the Bose-Einstein condensation if the phase-space density exceeds one. This phenomenon manifests itself by the macroscopic occupation of the single particle ground state. In the case of an interacting system the condensate wave function can be defined by spectral decomposition of the one-body reduced density matrix. This decomposition is closely related to the off-diagonal long range order [1, 4 ] and existence of the order parameter i.e. the 'classical' field with given amplitude and phase commonly used in the theory of superfluidity $[5,6$. 6 . Realization of decomposition procedure is practically impossible because it requires a full solution of the many-body problem. Instead mean-field approaches are commonly used. The basic idea for a mean-field description of the dilute, weakly interacting Bose gas below transition temperature was introduced by Bogoliubov [7]. Most of the results for the interacting Bose-Einstein condensate are obtained within the Bogoliubov theory which in many cases provides a reliable quantitative description of the quantum Bose gas. On the contrary, the rigorous treatment is possible only for model interactions.

There are only few exactly soluble models of quantum systems where the interactions between atoms is chosen in the form allowing for the exact analytic solution. These are: (i) the one-dimensional model of impenetrable bosons introduced by Girardeau [8], (ii) its contact potential version formulated by Lieb [9]; (iii) the model of particles interacting by harmonic forces [10 12]. Although in the first two cases the formal solution is given but in practice the problem is still quite complicated and quantitative calculations can be done for a very small number of particles only [13]. The latter case seems to be much simpler because, as it has been shown in [14, it can be reduced to the problem of noninteracting particles in a harmonic trap. Therefore in the following we are going to examine, within this exactly soluble model, various concepts related to the interacting Bose-Einstein condensate.

This paper is organized as follows. In Sec. II we present exact results for harmonically interacting bosons trapped within harmonic potential [14]. In Sec. III we find the analytic expression for the order parameter and study the effect of quantum depletion of the condensate as well as quantum fluctuations at zero temperature. In Sec. III we analyse the off-diagonal and diagonal order and show how off-diagonal long range order disappears when interactions become very strong. We finish in Sec. IV with some concluding remarks.

\section{DIAGONALIZATION OF THE HAMILTONIAN}

In our previous paper [14] we have shown the algebraic method of diagonalization of the Hamiltonian describing a system of many particles interacting via harmonic forces. The system under consideration consists of many particles confined by an external harmonic potential interacting by harmonic forces, i.e. two body interaction potential has the form: 


$$
V\left(\mathbf{x}_{i}-\mathbf{x}_{j}\right)=\frac{\sigma}{2} \Omega^{2}\left(\mathbf{x}_{i}-\mathbf{x}_{j}\right)^{2},
$$

where $\Omega$ defines the interaction strength and $\sigma=+1$ signifies the attractive interaction of particles placed at positions $\mathbf{x}_{i}$ and $\mathbf{x}_{j}$ whereas $\sigma=-1$ - corresponds to repulsive interactions. The total Hamiltonian of the $N$-particle system has therefore the following form:

$$
H=\sum_{i=1}^{N} \frac{1}{2}\left(\mathbf{p}_{i}^{2}+\mathbf{x}_{i}^{2}\right)+\sum_{i<j} V\left(\mathbf{x}_{i}-\mathbf{x}_{j}\right) .
$$

Let us first recall some exact results of [14]. For the sake of simplicity we denote the set of all particle positions vectors by $\mathbf{X}_{N}=\left(\mathbf{x}_{1}, \ldots, \mathbf{x}_{N}\right)$. The Hamiltonian which is a quadratic form of positions and momenta of particles can be easily diagonalized if one introduces collective variables:

$$
\mathbf{X}_{N}^{c}=\mathcal{Q}_{\mathcal{N}} \mathbf{X}_{N}
$$

where $\mathbf{X}_{N}^{c}=\left(\mathbf{x}_{1}^{c}, \ldots, \mathbf{x}_{N}^{c}\right)$ and the matrix $\mathcal{Q}_{N}=\left\{q_{i j}^{N}\right\}$ is orthogonal. One of these collective variables namely the center of mass of $N$-particle system plays a particularly important role:

$$
\mathbf{x}_{N}^{c}=\frac{1}{\sqrt{N}} \sum_{i=1}^{N} \mathbf{x}_{i}
$$

The choice of $N-1$ remaining variables $\mathbf{X}_{N-1}^{c}=\left(\mathbf{x}_{1}^{c}, \ldots, \mathbf{x}_{N-1}^{c}\right)$ is not unique but this does not lead to any physical implications. In particular:

$$
\left(\mathbf{X}_{N-1}^{c}\right)^{2}=\sum_{i=1}^{N-1}\left(\mathbf{x}_{i}^{c}\right)^{2}=\sum_{i=1}^{N}\left(\mathbf{x}_{i}\right)^{2}-\left(\mathbf{x}_{N}^{c}\right)^{2} .
$$

In the following we are going to use a similar notation for description of a subsystem of $s$-particles, $s=1, \ldots, N$.

The transformation defined above brings the Hamiltonian to the diagonal form and its eigenenergies can be easily found. However, while determining a spectrum one must also take into account the proper symmetry of a total wave function. In the case of bosonic particles $(N>2)$ the allowed energies are:

$$
E=\left(\frac{3}{2}+m\right)+\left(\frac{3}{2}(N-1)+n\right) \omega
$$

where $m=0,1,2 \ldots, n=0,2,3 \ldots$ and $\omega=\sqrt{1+\sigma N \Omega^{2}}$. The first term describes excitations of the center of mass - d-dimensional harmonic oscillator of frequency equal to one. The second term in the Eq.(6) corresponds to excitations of $N-1$ relative degrees of freedom. The frequency $\omega$ characterizes the effective potential experienced by $N-1$ collective modes.

Let us observe that $\omega=1$ corresponds to the noninteracting case, the repulsive interactions give $0<\omega<1$ while attractive forces lead to $\omega>1$. Moreover, very small values of $\omega \approx 0$ signify very strong repulsion which almost destabilizes the whole system. It is very convenient to parameterize $\omega$ by an exponent $\kappa$ defined in the following way:

$$
\omega=N^{\kappa} .
$$

This exponent can be related to the actual strength of the interaction. In fact, for weakly interacting gas $(\omega \approx 1)$ we obtain very small values of this parameter: $\kappa \approx 0$, while for strong interactions $(\omega \approx 0$ - repulsion, $\omega \gg 1-$ attraction) we have $|\kappa| \gg 1$. Moreover, $\kappa$ is positive in the case of attraction while it is negative for repulsion. Let us add at this point that in realistic situations of short-range interparticle interactions, large Bose-Einstein condensates can exist only for repulsive forces. In the case of attraction the size of the trapped condensate is limited to about 1500 atoms [5]. In our oscillatory model the forces between particles are negligible at small distances, therefore the model leads to the condensation (in the thermodynamic limit) in both attractive and repulsive case.

The ground state of the system is the following:

$$
\Psi\left(\mathbf{X}_{N}\right)=\Phi_{0}\left(\sqrt{\omega} \mathbf{X}_{N-1}^{c}\right) \Phi_{0}\left(\mathbf{x}_{N}^{c}\right),
$$


where $\left(\mathbf{X}_{N-1}^{c}, \mathbf{x}_{N}^{c}\right)=\mathcal{Q}_{N} \mathbf{X}_{N}$ and the function $\Phi_{0}\left(\sqrt{\omega} \mathbf{X}_{N-1}^{c}\right)$ corresponds to the ground state of a system of $N-1$ independent quasi-particles (in $d$ spatial dimensions) interacting with an external potential of the harmonic oscillator of frequency $\omega$ :

$$
\Phi_{0}\left(\sqrt{\omega} \mathbf{X}_{N-1}^{c}\right)=\left(\frac{\omega}{\pi}\right)^{d(N-1) / 4} \exp \left[-\omega\left(\mathbf{X}_{N-1}^{c}\right)^{2} / 2\right],
$$

and $\Phi_{0}\left(\mathbf{x}_{N}^{c}\right)$ is the ground state of the single particle (center of mass) trapped into harmonic potential:

$$
\Phi_{0}\left(\mathbf{x}_{N}^{c}\right)=\left(\frac{1}{\pi}\right)^{d / 4} \exp \left[-\left(\mathbf{x}_{N}^{c}\right)^{2} / 2\right]
$$

Construction of excited eigenstates is difficult because it is not easy to impose the desired symmetry on the wave function. This procedure was describe in details in 14.

\section{ORDER PARAMETER AND QUANTUM DEPLETION}

If the energy of the system (or equivalently the temperature) is sufficiently small we expect that the system forms a Bose-Einstein condensate. The BEC of the ideal gas manifests itself by a macroscopic occupation of the single particle ground state. In the case of interacting system it is not obvious what is this particular state which is 'macroscopically occupied'. The identification of the macroscopically occupied quantum state is equivalent to the definition of the order parameter - the single particle wave function which is inherently related to the Bose condensation. The condensate subsystem can be then quite accurately described by the $N_{0}$-fold product of the order parameter, where $N_{0} \simeq \mathcal{O}(N)$ is the occupation of the condensate.

In the following we use our model to demonstrate how to define the order parameter, occupation of the condensate, and its fluctuations. At zero temperature the system is in the ground state and one might naively expect that it is totally Bose condensated. However, the ground state of the $\mathrm{N}$-particle bosonic system is not equivalent to the Bose-Einstein condensate. Interactions can significantly deplete the condensate. We are going to show this effect in the most spectacular but also in relatively simple case of the zero temperature.

Let us now define the hierarchy of the reduced $s$-particle density matrices which can be conventionally obtained by averaging the density matrix of the total system of $N$ particles over the degrees of freedom of $N-s$ remaining particles. For a given $N$-particle quantum state $\Psi\left(\mathbf{X}_{N}\right)$ the corresponding $s$-particle reduced density matrix $\rho_{s}\left(\mathbf{X}_{s} ; \mathbf{Y}_{s}\right)$ is defined by:

$$
\rho_{s}\left(\mathbf{X}_{s} ; \mathbf{Y}_{s}\right)=\int \mathrm{d} \mathbf{R}_{N-s} \Psi^{*}\left(\mathbf{X}_{s}, \mathbf{R}_{N-s}\right) \Psi\left(\mathbf{Y}_{s}, \mathbf{R}_{N-s}\right) .
$$

We use previously defined shorthand notation for vectors in the configuration space of $s$-particles, for example $\mathbf{X}_{N}=$ $\left(\mathbf{X}_{s}, \mathbf{R}_{N-s}\right)$ and $\mathbf{X}_{s}=\left(\mathbf{x}_{1}, \ldots, \mathbf{x}_{s}\right)$. The reduced density matrix describes the subsystem of $s$-particles and can be directly related to different measurement processes. For the statistical description of the system one should first of all define the statistical density matrix by averaging all $N$-particle density matrices with the appropriate statistical weights depending on the ensemble. In general it is quite a complicated task but at zero temperature there is only one quantum state of the system and no statistical averaging is necessary.

The total wave function (or density matrix) carries all the information about the system. In real experiments however one does not probe simultaneously all the particles. Typical detection scheme consists on the measurement of one or at most few particles at a given time. In other words a single measurement process is reduced to a subsystem of small number of particles. Such subsystems are described by reduced density matrices. In the considered here case of zero temperature the $s$-particle density matrix can be brought to the following form:

$$
\rho_{s}\left(\mathbf{X}_{s} ; \mathbf{Y}_{s}\right)=\rho^{C M}\left(\mathbf{x}_{s}^{c}, \mathbf{y}_{s}^{c}\right) \Phi_{0}\left(\sqrt{\omega} \mathbf{X}_{s-1}^{c}\right) \Phi_{0}\left(\sqrt{\omega} \mathbf{Y}_{s-1}^{c}\right)
$$

The functions $\Phi_{0}\left(\sqrt{\omega} \mathbf{X}_{s-1}^{c}\right)$ describes the ground state of $s-1$ quasi-particles:

$$
\Phi_{0}\left(\sqrt{\omega} \mathbf{X}_{s-1}^{c}\right)=\left(\frac{\omega}{\pi}\right)^{d(s-1) / 4} \exp \left[-\omega\left(\mathbf{X}_{s-1}^{c}\right)^{2} / 2\right]
$$

while $\rho^{C M}\left(\mathbf{x}_{s}^{c}, \mathbf{y}_{s}^{c}\right)$ corresponds to the density matrix of center of mass of the subsystem: 


$$
\rho^{C M}\left(\mathbf{x}_{s}^{c}, \mathbf{y}_{s}^{c}\right)=\left(\frac{\omega_{s}}{\pi}\right)^{d / 2} \exp \left\{-\frac{1}{2}\left(\omega_{s}+\frac{\delta_{s}}{2}\right)\left[\left(\mathbf{x}_{s}^{c}\right)^{2}+\left(\mathbf{y}_{s}^{c}\right)^{2}\right]+\frac{\delta_{s}}{2} \mathbf{x}_{s}^{c} \mathbf{y}_{s}^{c}\right\} .
$$

The $s$-particles collective coordinates are defined in the familiar way: $\left(\mathbf{X}_{s-1}^{c}, \mathbf{x}_{s}^{c}\right)=\mathcal{Q}_{s} \mathbf{X}_{s},\left(\mathbf{Y}_{s-1}^{c}, \mathbf{y}_{s}^{c}\right)=\mathcal{Q}_{s} \mathbf{Y}_{s}$ and frequencies $\omega_{s}, \delta_{s}$ as well as auxiliary parameter $\gamma_{s}$ are:

$$
\begin{aligned}
\gamma_{s} & =1-\frac{s(1-\omega)}{N} \\
\omega_{s} & =\frac{\omega}{\gamma_{s}} \\
\delta_{s} & =\left(\frac{1-\omega}{N}\right)^{2} \frac{s(N-s)}{\gamma_{s}}
\end{aligned}
$$

Having defined the $s$-particle matrices we are ready now to analyze the nature of the Bose-Einstein condensation of the interacting system and to discuss the meaning of the order parameter. To this end we write the density matrix Eq.(12) in the diagonal form:

$$
\rho_{s}\left(\mathbf{X}_{s} ; \mathbf{Y}_{s}\right)=\sum_{\mathbf{n}} \lambda_{\mathbf{n}}^{(s)} \phi_{\mathbf{n}}^{(s)}\left(\mathbf{X}_{s}\right) \phi_{\mathbf{n}}^{(s)}\left(\mathbf{Y}_{s}\right)
$$

The function $\phi_{\mathbf{n}}^{(s)}\left(\mathbf{X}_{s}\right)$ can be treated as the wave function of the $s$-particle subsystem:

$$
\phi_{\mathbf{n}}^{(s)}\left(\mathbf{X}_{s}\right)=\Phi_{0}\left(\sqrt{\omega} \mathbf{X}_{s-1}^{c}\right) \Phi_{\mathbf{n}}\left(\sqrt{\alpha_{s}} \mathbf{x}_{s}^{c}\right)
$$

where $\Phi_{0}\left(\sqrt{\omega} \mathbf{X}_{s-1}^{c}\right)$ is the ground state wave function of the relative degrees of freedom. This function corresponds to the ground state of $s-1$ noninteracting quasi-particles (in d-spatial dimensions) subject to the external harmonic potential of frequency $\omega$. The second part of the Eq. 19 describes states of the center of mass of $s$-particles; $\Phi_{\mathbf{n}}$ is simply the d-dimensional harmonic oscillator wave function corresponding to the effective center of mass frequency $\alpha_{s}$. Quantum numbers $\mathbf{n}=\left(n_{1}, \ldots, n_{d}\right)$ label different states of the center of mass while $n=n_{1}+\ldots+n_{d}$ corresponds to the energy of the given state. The effective center of mass frequency $\alpha_{s}$ is:

$$
\alpha_{s}=\left[\omega_{s}\left(\omega_{s}+\delta_{s}\right)\right]^{1 / 2}
$$

It is interesting to observe that all the frequencies of the relative motion of the $s$-particles subsystem are exactly the same as the frequencies of the relative motion of the whole system, i.e. equal to $\omega$. On the other hand the center of mass oscillation frequency of the subsystem is neither equal to $\omega$ nor to 1 (trap frequency). This collective degree of freedom couples to the center of mass of $N-s$ remaining particles what leads to some energy shift. Finally, the eigenvalues $\lambda_{n}^{(s)}$ of $\rho_{s}$ are equal to the occupation probabilities of a given $s$-particle state:

$$
\lambda_{n}^{(s)}=\left(\frac{\omega_{s}}{\alpha_{s}}\right)^{d / 2}\left(\frac{2 \sqrt{\omega_{s} \alpha_{s}}}{\omega_{s}+\alpha_{s}}\right)^{d}\left(\frac{\alpha_{s}-\omega_{s}}{\alpha_{s}+\omega_{s}}\right)^{n}
$$

It follows from the normalization condition for the density matrix that: $\sum_{\mathbf{n}} \lambda_{n}^{(s)}=1$.

The spectral decomposition of the reduced one-particle density matrix gives natural single-particle states $\phi_{\mathbf{n}}^{(1)}(\mathbf{x})$. These states are crucial for the definition of the condensate wave function (order parameter). It can be seen from Eq.(21) that if $N$ goes to infinity (thermodynamic limit) with fixed value of the interaction strength $\kappa$ the lowest eigenvalue $\lambda_{0}^{(1)}$ dominates the others and in the limit of weak interactions we get:

$$
\begin{aligned}
& \lambda_{0}^{(1)} \simeq 1, \\
& \lambda_{\mathbf{n}}^{(1)} \simeq\left(\frac{(1-\omega)^{2}}{4 N}\right)^{n}, \quad \text { if } \quad n \neq 0 .
\end{aligned}
$$

This behavior signifies nothing else but the onset of the Bose-Einstein condensation. The single particle density matrix becomes very close to the pure state because with quite good accuracy it can be approximated by $\rho_{1}(\mathbf{x}, \mathbf{y}) \approx$ $\phi_{0}^{(1)}(\mathbf{x}) \phi_{0}^{(1)}(\mathbf{y})$. This particular single-particle ground state $\phi_{0}^{(1)}(\mathbf{x})$ is usually called the order parameter. The $N$ particle wave function can be quite accurately approximated by the $N$-fold product of the order parameter. 
Our analytic formula allows to study quantitatively the role of interactions on the Bose-Einstein condensate. On the basis of the discussion it is obvious that the average occupation of the condensate becomes:

$$
\left\langle N_{0}\right\rangle=N \int \mathrm{d} \mathbf{x} \mathrm{d} \mathbf{y} \phi_{0}^{(1)}(\mathbf{x}) \rho_{1}(\mathbf{x}, \mathbf{y}) \phi_{0}^{(1)}(\mathbf{y})=N \lambda_{0}^{(1)} .
$$

In the case of the ideal gas at zero temperature the above equation gives, of course, $\left\langle N_{0}\right\rangle=N$; all particles occupy the single particle ground state. For studied here model of the interacting system it is more convenient to use as independent variables the pair of $\kappa$ and $N$ rather than $\omega$ and $N$. The exponent $\kappa$ occurs to be inherently related to the oscillatory interactions because when using this parameter we discover some universal features. For a fixed number of particles, if the interaction strength $|\kappa| \sim \log \omega$ grows, the occupation of the condensate decreases. This behavior is presented in Fig. 1 where we show the mean occupation of the condensate versus the exponent $\kappa=\log \omega / \log N$ for different values of particle number $N$ in three spatial dimensions $(d=3)$. The values of $\kappa$ less than zero signify repulsive interactions while $\kappa>0$ corresponds to attraction. One can easily see that if the interaction becomes strong $(|\kappa| \simeq 1)$ the condensate is almost totally depleted. All curves presented in the figure tend to an universal curve if the number of particles increases. When $N$ increases to infinity with $\kappa$ being constant then our expression for the occupation of the condensate has the form:

$$
\frac{\left\langle N_{0}\right\rangle}{N}=\left(\frac{2}{1+\sqrt{N^{\kappa-1}+N^{-(\kappa+1)}+1}}\right)^{d} .
$$

The above formula, valid in the thermodynamic limit, gives an universal critical behavior. It exhibits no depletion $\left(N_{0}=N\right)$ for $|\kappa|<1$ followed by an abrupt jump and total destruction of the condensate $\left(N_{0}=0\right)$ for $|\kappa|>1$.

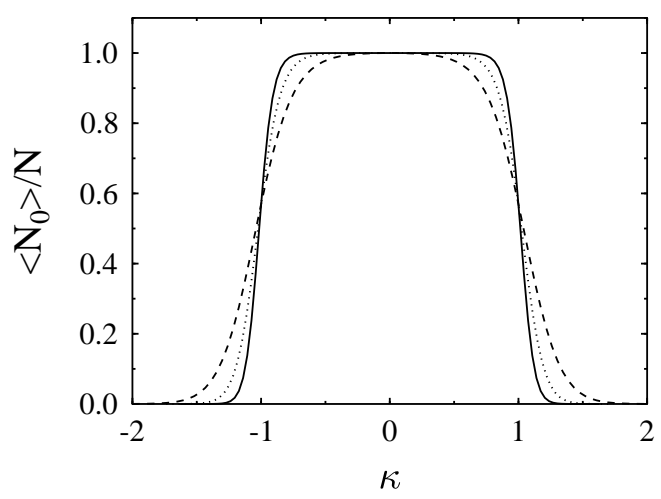

FIG. 1. Mean occupation of the condensate plotted as a function of the parameter $\kappa=\log \omega / \log N$ for different number of particles; $N=10^{3}$ - dashed line; $N=10^{5}$ - dotted line, and $N=10^{8}$ - full line.

The effect of quantum depletion of the trapped atomic condensate with a short range interactions, for the realistic experimental parameters, has been estimated to be of the order of $1 \%$.57. This is opposite to the case of superfluid helium where this effect accounts for depletion as big as more than $90 \%$ [15]. Our model exhibits very interesting feature. It shows that in large $N$ limit the quantum effects are almost negligible or totally destroy the condensate depending on the value of the interaction strength. At this point it is not clear if this is an unique feature of our model or if it is more general result.

The 2-particle reduced density matrix allows to find a joint probability of finding a particle in a given single particle state provided that another particle is also in some given state. In particular we have:

$$
\left\langle N_{0}\left(N_{0}-1\right)\right\rangle=N(N-1) \int \mathrm{d} \mathbf{X}_{2} \mathrm{~d} \mathbf{Y}_{2}\left[\phi_{0}^{(1)}\left(\mathbf{x}_{1}\right) \phi_{0}^{(1)}\left(\mathbf{x}_{2}\right)\right] \rho_{2}\left(\mathbf{X}_{2}, \mathbf{Y}_{2}\right)\left[\phi_{0}^{(1)}\left(\mathbf{y}_{2}\right) \phi_{0}^{(1)}\left(\mathbf{y}_{1}\right)\right] .
$$

Simple integration gives:

$$
\left\langle N_{0}\left(N_{0}-1\right)\right\rangle=N(N-1)\left(\frac{2 \sqrt{\omega \alpha_{1}}}{\omega+\alpha_{1}}\right)^{d}\left(\frac{2 \sqrt{\omega_{2} \alpha_{1}}}{\omega_{2}+\alpha_{1}}\right)^{d}\left(\frac{\omega_{2}+\alpha_{1}}{\omega_{2}+\alpha_{1}+\delta_{2}}\right)^{d / 2} .
$$


Now we are ready to analyze the fluctuations of the condensate defined as:

$$
\left\langle\delta^{2} N_{0}\right\rangle=\left\langle\left(N_{0}\right)^{2}\right\rangle-\left\langle N_{0}\right\rangle^{2} .
$$

These fluctuations are shown in Fig.2. We see that as the interaction strength grows (at fixed number of particles) the fluctuations start to grow from zero value for the ideal gas. However, again when the interactions become so strong that condensate practically disappears $(|\kappa| \simeq 1)$ fluctuations also decrease - as there is no condensate the fluctuations also die out. The fluctuations are maximal in a region of the critical destruction of the condensate by quantum effects.

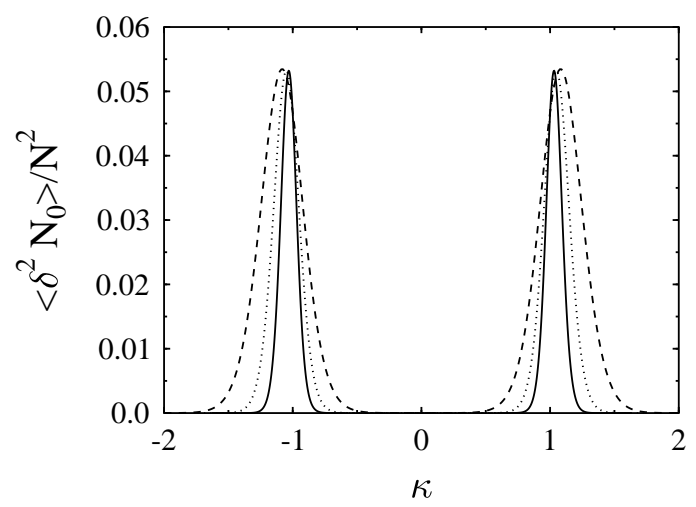

FIG. 2. Fluctuations of the condensate plotted as a function of the parameter $\kappa=\log \omega / \log N$ for different number of particles; $N=10^{3}$ - dashed line; $N=10^{5}$ - dotted line, and $N=10^{8}$ - full line.

\section{OFF-DIAGONAL ORDER AND CORRELATIONS}

In this subsection we are going to study (within our model) the phase and density correlations for the ground state of the interacting system of bosonic particles. In the early works of Penrose and Onsager [i] it has been shown that phenomenon of Bose condensation is evidenced by the presence of the off-diagonal long range order in the one-particle density matrix $\rho_{1}(\mathbf{x}, \mathbf{y})$ :

$$
\lim _{|\mathbf{x}-\mathbf{y}| \rightarrow \infty} \rho_{1}(\mathbf{x}, \mathbf{y}) \neq 0
$$

The above condition signifies a large scale correlations of the off-diagonal elements of the single-particle matrix. The limiting value of correlations is called the off-diagonal long range order parameter. In a finite system this concept cannot be strictly applied as the reduced density matrix vanishes at large distances. However, for a practical purposes the off-diagonal long range order can be related to the behavior of the off-diagonal elements of the density matrix at distances equal to the size of the system.

In order to identify long range correlations we should first analyze characteristic length scales. It is natural to relate the size of the system to the spatial extension of the single particle density, i.e. the diagonal elements of the one-particle matrix:

$$
n_{1}(\mathbf{x})=N \rho_{1}(\mathbf{x}, \mathbf{x})=N\left(\frac{\omega_{1}}{\pi}\right)^{d / 2} \exp \left(-\omega_{1} \mathbf{x}^{2}\right) .
$$

Therefore, the size of our system is:

$$
\ell_{1}=\frac{1}{\sqrt{\omega_{1}}}
$$

For large values of $N$ an extension of the system for attractive interactions approaches the value of one, $\ell_{1} \approx 1$, i.e. is governed by the characteristic length scale of the external potential felt by the center of mass. Relative degrees of freedom are localized within much smaller distances $1 / \sqrt{\omega} \ll \ell_{1}$. For repulsive interactions a spatial extension of the system is related to the length scale of a mean field potential experienced by relative degrees of freedom, $\ell_{1} \approx 1 / \sqrt{\omega}$. In this case the magnitude of the center of mass spreading is relatively small $1 \ll \ell_{1}$. These features have serious 
implications on behavior of the order parameter and off-diagonal order. Let us stress that existence of different length scales - the one related to the external potential, and another related to the interparticle interactions is the general feature of all two-body central forces. This is not an unique behavior of considered here long range oscillatory model.

The presence of the off-diagonal long range order in the case of inhomogeneous system of finite spatial extension manifests itself by a large distance behavior of the following correlation function:

$$
g_{1}(\mathbf{x}, \mathbf{y})=\frac{N \rho_{1}(\mathbf{x}, \mathbf{y})}{\sqrt{n_{1}(\mathbf{x}) n_{1}(\mathbf{y})}} .
$$

Accordingly, we shall refer to notion of the off-diagonal long range order if:

$$
\lim _{|\mathbf{x}-\mathbf{y}| \rightarrow \ell_{1}} g_{1}(\mathbf{x}, \mathbf{y}) \neq 0
$$

By dividing the correlation function by the square root of single particle densities we ensure that correlations are not sensitive to the local density of particles. Let us observe that off-diagonal correlation function describes phase correlations in our system. In the case when the one-particle density matrix corresponds to a pure state $\Psi(\mathbf{x})=$ $R(\mathbf{x}) \exp [\mathrm{i} S(\mathbf{x})]$ (where $R(\mathbf{x})$ and $S(\mathbf{x})$ are the modulus and phase of the wave function), then $g_{1}(\mathbf{x}, \mathbf{y})=\exp \{-\mathrm{i}[S(\mathbf{x})-$ $S(\mathbf{y})]\}$. In the context of the Bose-Einstein condensation of trapped alkali gases such phase correlations in a trapped 2-dimensional interacting system has been studied recently in Ref. [16].

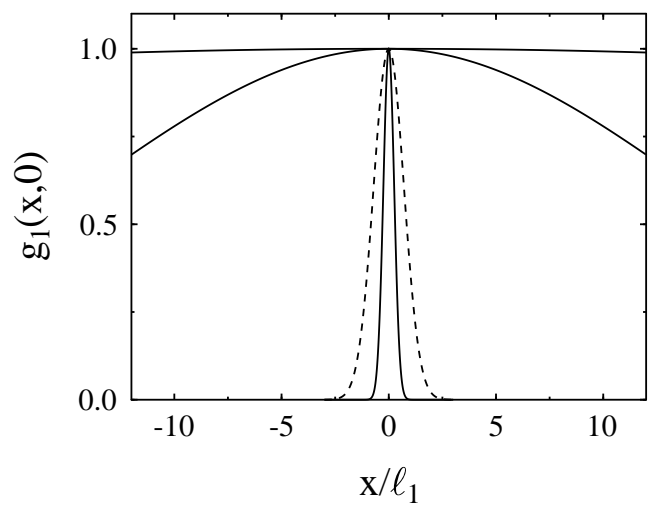

FIG. 3. Off-diagonal correlations $\left.g_{1}(x, 0)\right)$ for a system of $N=10^{5}$ particles for different interaction strength $\kappa=\log \omega / \log N$. Note that $g_{1}(x, 0)$ does not depend on the sign of interaction. The full lines correspond to the following values of $|\kappa|$ (from the top to the bottom); $|\kappa|=0.3,0.6,1.3$. The dashed line corresponds to the single particle density function $n_{1}(x) / N$. The distance has been scaled in such a way that single particle density does not depend on the strength of interaction.

Now we are going to apply the above definition to study the coherence of the ground state of bosonic particles interacting by harmonic forces. Similarly as in the previous section we will investigate the large $N$ limit varying the interaction strength $\kappa$. Because the system is inhomogeneous the correlation function depends on the six variables (in three dimensional space). To simplify our analysis we fix the position $\mathbf{y}$ at the trap center, $\mathbf{y}=0$. With this restriction the correlation function depends only on the distance $x=|\mathbf{x}|$ :

$$
g_{1}(x, 0)=\exp \left(-\frac{\delta_{1}}{4} x^{2}\right),
$$

what gives for the limit of large distances, $x \sim \ell_{1}$, the expression for the off-diagonal order:

$$
g_{1}\left(\ell_{1}, 0\right)=\exp \left(-\frac{\delta_{1}}{4 \omega_{1}}\right) \text {. }
$$

In the Fig.3 we show the behavior of the off-diagonal order Eq. 34) as a function of distance to the trap center for $N=10^{5}$ particles and different interaction strength $\kappa$. We see that for a weak interaction the system exhibits the off-diagonal long range order, while if interactions are strong the phase correlations rapidly vanish at distances smaller 
than the natural size of the system. For comparison we show in the Fig.3 the single particle density $n(x) / N($ dashed line). This kind of dependence of the off-diagonal order is consistent with the behavior of the order parameter (the condensate wave function) discussed previously. Indeed, the coherence length $L_{o f f}^{(1)}$ which appears in the equation Eq.(35) in the large $N$ limit has a form:

$$
L_{o f f}^{(1)}=\left(\frac{\omega_{1}}{\delta_{1}}\right)^{1 / 2}=\frac{1}{\sqrt{N^{-\kappa-1}+N^{\kappa-1}}} .
$$

Let us notice that the coherence length is governed by the same type of $\kappa$-dependence as the mean occupation of the condensate Eq.(25). Therefore it is not surprising that the order parameter and off-diagonal order behaves similarly. Disappearing of the off-diagonal long range order has exactly the same critical character as destruction off the order parameter when the interaction strength exceeds value one, $|\kappa|>1$. The coherence length in large $N$ limit, $N \rightarrow \infty$ is:

$$
\begin{array}{lll}
L_{o f f}^{(1)}=\infty, & \text { if } & |\kappa|<1, \\
L_{o f f}^{(1)}=0, & \text { if } & |\kappa|>1 .
\end{array}
$$

So far we have studied the off-diagonal correlations for the one-particle reduced matrix. However, a similar analysis can be done for any $s$-particle density matrix. Without going into unnecessary algebra one can easily estimate the coherence length for a given $s$-particle subsystem. The form of the corresponding reduced density matrix (see Eq. 144) explicitly suggests that the order of magnitude of the off-diagonal elements at distances comparable to the size of the system, $x=\ell_{1}$ can be estimated by: $\exp \left[-\delta_{s} /\left(4 \omega_{s}\right)\left(x / \ell_{1}\right)^{2}\right]$. Therefore, the $s$-order coherence length is:

$$
L_{o f f}^{(s)}=\left(\frac{\omega_{s}}{\delta_{s}}\right)^{1 / 2}=\frac{1}{\sqrt{s\left(N^{-\kappa-1}+N^{\kappa-1}\right)}} .
$$

This expression shows that there is an off-diagonal long range coherence on each level of hierarchy of the reduced density matrices provided that $|\kappa|<1$.

This statement, however, requires some comment. In fact the equation Eq. (39) is valid only if $\lim _{N \rightarrow \infty}(s / N)=0$, i.e. if we consider the subsystem of finite number of particles (while the total number of particles is infinite). On the contrary, in the $\operatorname{limit}_{\lim _{N \rightarrow \infty}}(s / N)=\beta>0$ we have:

$$
L_{o f f}^{(\beta)}=\left(\frac{\omega_{s}}{\delta_{s}}\right)^{1 / 2}=\frac{1}{\sqrt{\beta(1-\beta)\left(N^{-\kappa}+N^{\kappa}\right)}} \longrightarrow 0 .
$$

There is no coherence in large subsystems when the number of particles in the subsystem is of the order of the total number of particles, $s \sim \mathcal{O}(N)$. However, as long as the detection scheme is reduced to an observation of at most few particles simultaneously (what is true for all experiments) the Bose system at zero temperature can be viewed as described by the coherent state of many particles. This point of view is justified on every level of the reduced $s$-particle density matrix provided that $s / N \ll 1$. This kind of behavior is in a spirit of Yang ( [3]) conjecture, who suggested that Bose-Einstein condensation is evidenced by the fact that every $s$-particle matrix can be related to a simple $s$-fold product of the order parameter. In our model of the interacting condensate the above statement is true for a whole hierarchy of $s$-particle matrices when $s / N \rightarrow 0$. If $s / N \ll 1$ and $|\kappa|<1$ simple integration gives:

$$
\rho_{s}\left(\mathbf{X}_{s}, \mathbf{Y}_{s}\right) \simeq \beta^{(s)}\left[\Phi_{0}^{(1)}\left(\mathbf{x}_{1}\right) \ldots \Phi_{0}^{(1)}\left(\mathbf{x}_{s}\right)\right]\left[\Phi_{0}^{(1)}\left(\mathbf{y}_{1}\right) \ldots \Phi_{0}^{(1)}\left(\mathbf{y}_{s}\right)\right]
$$

where $\beta^{(s)}$ is:

$$
\beta^{(s)}=\left(\frac{2 \sqrt{\omega \alpha_{1}}}{\omega+\alpha_{1}}\right)^{d}\left(\frac{2 \sqrt{\omega_{s} \alpha_{1}}}{\omega_{s}+\alpha_{1}}\right)^{d}\left(\frac{\omega_{s}+\alpha_{1}}{\omega_{s}+\alpha_{1}+\delta_{s}}\right)^{d / 2} \longrightarrow 1
$$

The equation Eq.(41) is not valid if one considers $s$-particle matrices for $s$ being of the order of the total number of particles, $s \sim \mathcal{O}(N)$. At this point we want to make a comment about the notion of the coherence of the Bose-Einstein condensate. Approximate methods assume explicitly that a mean value of the boson field operator is different than zero in the case of the Bose-Einstein condensate. Therefore, the folk wisdom associates the condensate with the coherent state - the analogue of the coherent state of the electromagnetic field. This analogy is of limited value and 
in fact may be misleading because the condensate must be in a Fock state in which a mean value the field operator vanishes. However, there is coherence in the condensate in the sense that majority of particles is described by the same wave function with the same phase. The coherence of the condensate manifests itself by the off-diagonal long range order. The coherence length of a phase of the condensate is at least of the same magnitude as the spatial extension of the system. Moreover this coherence is present also when one considers the subsystems of any number of particles significantly smaller than a total particle number. This facts allows for substantial simplification of description of many particle system. When the system is coherent, i.e. when one can assign exactly the same wave function to every particle then a behavior of the condensate can be correctly described by this single-particle function. The above does not apply to measurements based on simultaneous detection of almost all particles. In such a case the $N$-body wave function has to be used instead.

The presented analysis of the order parameter and off-diagonal correlations exposed a kind of symmetry with respect to change of the interaction sign. The differences can be seen while studying the diagonal correlations of the reduced density matrices. The diagonal elements of one-particle matrix are related to single particle density, $n_{1}(\mathbf{x})=N \rho_{1}(\mathbf{x}, \mathbf{x})$ while $\rho_{2}(\mathbf{x}, \mathbf{y} ; \mathbf{x}, \mathbf{y})$ describes a joint probability of finding a particle at position $\mathbf{x}$ provided than another particle is at position $\mathbf{y}$. The corresponding two-particle density (if $\mathbf{x} \neq \mathbf{y}$ ) is:

$$
n_{2}(\mathbf{x}, \mathbf{y})=N(N-1) \rho_{2}(\mathbf{x}, \mathbf{y} ; \mathbf{x}, \mathbf{y}) .
$$

The above function can be related to two-particle correlation function:

$$
g_{2}(\mathbf{x}, \mathbf{y})=\frac{n_{2}(\mathbf{x}, \mathbf{y})-n_{1}(\mathbf{x}) n_{1}(\mathbf{y})}{n_{1}(\mathbf{x}) n_{1}(\mathbf{y})}
$$

If two-particle density can be written as a product of corresponding one-particle ones then there are no diagonal (density) correlations, i.e. $g_{2}(\mathbf{x}, \mathbf{y})=0$. Positive values of $g_{2}$ signify some clustering effect while negative values correspond to some effective repulsion. Note that $g_{2}$ can be in principle infinitely large (extremely strong attraction) but its minimal value is limited by -1 . This value corresponds to extremely strong repulsion when particles tend to avoid each other.

In the case of studied here model, all density functions are inhomogeneous. Therefore, for the sake of simplicity we again fix one of the position vectors at the trap center, i.e. we set $\mathbf{y}=0$. Then the correlation function $g_{2}$ depends only on the distance $x=|\mathbf{x}|$ :

$$
g_{2}(x, 0)=\frac{N-1}{N}\left(\frac{\omega \omega_{2}}{\omega_{1}^{2}}\right)^{d / 2} \exp \left[-\frac{1}{2}\left(\omega+\omega_{2}-2 \omega_{1}\right) x^{2}\right]-1 .
$$

The above equation gives the characteristic length scales of two-particle diagonal correlation:

$$
L_{\text {diag }}^{(2)}=\left(\frac{\omega_{1}}{\omega+\omega_{2}-2 \omega_{1}}\right)^{1 / 2}
$$

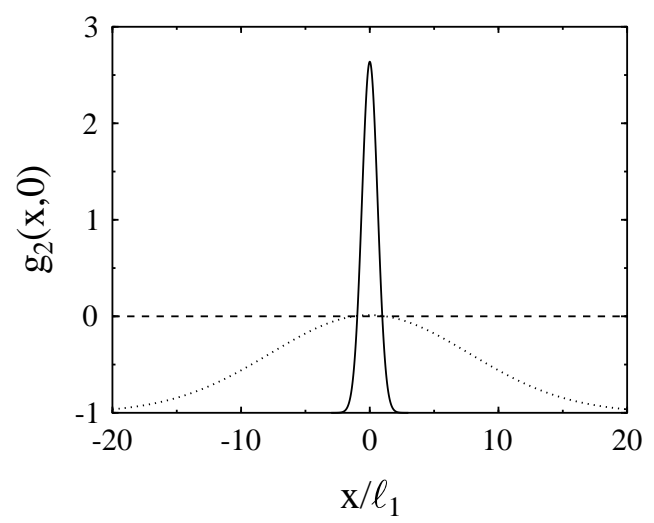

FIG. 4. Diagonal correlation $\left.g_{2}(x, 0)\right)$ for a system of $N=10^{5}$ particles for different interaction strength $\kappa=\log \omega / \log N$; $\kappa=0.4$ - dashed line; $\kappa=0.8$ - dotted line; $\kappa=1.1$ - full line. 
Let us first consider the attractive forces. In this case the diagonal correlation function strongly depends on the magnitude of the interaction strength $|\kappa|$. For weak interactions, $0<\kappa<1$, the diagonal correlation length becomes very large $L^{(2)} \rightarrow \infty$ similarly as the off-diagonal one. This is however, not necessarily the signature of strong correlations in the system. In the considered case the magnitude of correlations vanishes in large $N$ limit within the whole system, $g_{2}(x, 0) \approx 0$ if $|x|<\ell_{1}$. If attraction becomes very strong, $\kappa>1$, then the diagonal correlation length drastically decreases:

$$
L_{\text {diag }}^{(2)} \approx 1 / \sqrt{N^{\kappa-1}}=L_{\text {off }}^{(2)} \rightarrow 0 .
$$

In fact it becomes equal to the phase coherence length. Simultaneously the magnitude of these diagonal correlations grows to infinity, $g_{2}(x, 0) \approx\left(N^{\kappa-1}\right)^{d / 2}$ if $|x|<\ell_{1}$. This short range diagonal order is related to the clustering of particles. The joint probability of finding two particles very close to each other is very large provided that their separation is smaller than $L_{\text {diag }}^{(2)}$. Although the particles are clustered the position of this 'clustering spot' is localized within the large distance determined by the spatial extension of the center of mass ground state, $L_{\text {diag }}^{(2)} \ll \ell_{1} \approx 1$. This behavior is illustrated in Fig.4. The detailed comparison with corresponding off-diagonal correlations, Fig. 3 is very interesting. The presence off the off-diagonal long range order in the system is accompanied by the absence of the diagonal order for relatively weak interactions, $\kappa<1$. If interaction becomes very strong, $\kappa>1$, then a diagonal order becomes very large on a very small distance. Simultaneously the off-diagonal order disappears. Both characteristic length scales for diagonal and off-diagonal correlations are the same.

For the repulsive forces the diagonal order depends very weakly on the interaction strength. The magnitude of diagonal correlations tends to zero with increasing particle number, $g_{2}(x, 0) \approx 2 N^{-1}-3 N^{\kappa} \rightarrow 0$ while the correlation length is large $L_{\text {diag }}^{(2)}=N / \sqrt{1-2 N^{\kappa}+2 N^{-1}} \rightarrow \infty$. The change of the behavior of the diagonal order with the interaction strength $\kappa$ is similar to the case of attractive forces (see Fig.3). However, as the correlations are practically negligible a small diagonal order which appears when $\kappa<-1$ is almost unvisible. In general we can say that similarly as in the case of attraction, there are no diagonal correlations when the system exhibits the off-diagonal long range order.

\section{CONCLUSIONS}

In our paper we used exactly soluble many-particle model to illustrate a rigorous procedure of definig the condensate phase at zero temperature. By diagonalizing one-particle reduced density matrix we were able to study in details the role of interactions on the condensate. If the interaction strength becomes large $|\kappa|>1$ the condensate disappears even when the system is in its ground state. In the oscillatory model studied here this total depletion of the condensate has a critical character in the thermodynamic limit.

The existence of nonvanishing order parameter is accompanied by the off-diagonal long range order. The off-diagonal order is present on all levels of the hierarchy of reduced $s$-particle density matrices $(s / N \ll 1)$ as long as $|\kappa|<1$. There is no diagonal long range order if the system exhibits the coherence (off-diagonal order).

All these results have been obtained in the framework of a model. The model has some unrealistic features, its advantege is that it is exactly soluble. Therefore all the results presented in the paper are rigorous within the model.

Most of the results are, however, model-independent and in fact were discussed previously but without rigorous proof. Thus we provided another way of justifying them by showing that they can be proved for model interactions.

In particular the existence of (at least) two diffeent length scales, the one related to the external potential and the other resulting from the combined effect of interparticle interactions is a generic feature of all interacting systems. Moreover, as the first scale does not depend on the number of particles and interaction strength the second one does. The last scale is related to the spatial extention of the relative degrees of freedom. Our model shows that there is a coherence in the system if the two length scales are of the same order (different collective eigenmodes are not spatially separated). However, when these two scales become drastically differrent there is a spatial separation of different phases and off-diagonal long range order is destroyed.

In the case of attraction the effect of destruction of the condensate relays on the fact that particles cluster in the region of the size much smaller than the center of mass range. On the contrary, for repulsive forces the condensate disapears when the amplitude of the center of mass zero-point oscillations is much smaller than the size of the system. In fact in the case of realistic attractive forces it is well known that interactions lead to clustering of atoms. Therefore condensates with negative scattering length are limited to relatively small number of atoms [5]. Our results suggest however, that there is a similar limitation on the size of the condensate interacting by repulsive forces. In fact in the 
case of short range forces the Thomas-Fermi approach gives the ratio of the condensate size, $R$, to the characteristic length scale, $a_{h o}$, of the trap potential in the form: $R / a_{h o}=\left(15 N a_{s} / a_{h o}\right)^{1 / 5}$ whre $a_{s}$ is the scattering length. For the largest existing trapped condensates $\left(N \sim 10^{7}\right)$ this value is about 10 . This somehow fits perfectly to the scenario of destruction of the condensate by interactions presented in this paper.

\section{ACKNOWLEDGEMENT}

This work was supported by the KBN grant 2 P03B 13015 .

[1] O. Penrose, Phyl. Mag. 42, 1373 (1951); O. Penrose, L. Onsager, Phys. Rev. 104, 576 (1956).

[2] V.L. Ginzburg, L.D. Landau, Zh. Eksp. Teor. Fiz. 20, 1064 (1950).

[3] C.N. Yang, Rev. Mod. Phys. 34, 694 (1962).

[4] R.M. Ziff, G.E. Uhlenbeck, M. Kac, Phys. Rep. 32C, 171 (1977).

[5] F. Dalfovo, S. Giorgini, L. Pitaevskii, S. Stringari, Rev. Mod. Phys. 71, 463 (1999).

[6] A. Griffin, Excitations in a Bose-Condensed Liqiud (Cambridge University Press, New York, 1993).

[7] N. Bogoliubov, J. Phys. USSR 11, 23 (1947).

[8] M. Girardeau, J. Math. Phys. 1, 516 (1960)

[9] E.H. Lieb, W. Liniger, Phys. Rev. 130, 1605 (1963); E.H. Lieb, Phys. Rev. 130, 1616 (1963).

[10] F. Calogero, J. Math. Phys. 10, 2191 (1969); J. Math. Phys. 10, 2197 (1969); J. Math. Phys. 12, 419 (1971).

[11] This kind of interactions has been used in various context, see for example S. Gartenhaus, C. Schwartz, Phys. Rev. 108, 482 (1957); I. Białynicki-Birula, Lett. Math. Phys. 10, 189 (1985).

[12] The model of particles interacting by harmonic forces has been intensively studied recently in the context of trapped Bose and Fermi gases by L.F. Lemmens, F. Brosens, J.T. Devreese and collaborators, see for example L.F. Lemmens, F. Brosens, J.T. Devreese, Phys. Rev. A 59, 3112 (1999), S. Foulon, F. Brosens, J.T. Devreese, L.F. Lemmens, Phys. Rev. E 59, 3911 (1999).

[13] K. Góral, M. Wilkens, K. Rzążewski, submitted to A. J. Phys.

[14] M. Załuska-Kotur, M. Gajda, A. Orłowski, and J .Mostowski, Phys. Rev. A61, 033631 (2000).

[15] K. Huang, Statistical Mechanics (Wiley, New York, 1987).

[16] D.S. Petrov, M. Holtzmann, G.V. Shlyapnikov, Phys. Rev. Lett. 84, 2551 (2000). 